Revista Eletrônica de Farmácia

Eletronic Journal of Pharmacy

ISSN 1808-0804

doi $10.5216 /$ ref.v16ie.47986

Artigo Original

\title{
Perfil de utilização de medicamentos e substâncias psicoativas por alunos do ensino médio da escola de aplicação da UFPA
}

\section{Use of medicines and psychoactive substances by students of high school in UFPA application school}

\section{Perfil de utilización de medicamentos y sustancias psicoacti- vas por alumnos de nivel secundario de la escuela de aplicación de la UFPA}

\author{
BOTELHO, Johnathan Lucas da Silva ${ }^{1}$. FREITAS, Denilson Barbosa de ${ }^{1}$. ALMEIDA, Eduardo Dias ${ }^{1}$. BAPTISTA, Ester \\ Roseli ${ }^{1}$. SOLER, Orenzio ${ }^{1 *}$. \\ ${ }^{1}$ Faculdade de Farmácia, Instituto de Ciências da Saúde, Universidade Federal do Pará - ICS/UFPA. \\ *orenziosoler@ufpa.br
}

Resumo. Introdução: A adolescência é uma fase de desenvolvimento a qual tende a ocorrer os primeiros contatos com o uso de medicamentos psicoativos, bebidas alcoólicas e outras drogas. Objetivo: O objetivo desta investigação foi conhecer o perfil de utilização de medicamentos e de substâncias psicoativas em adolescentes do ensino médio matriculados na Escola de Aplicação da Universidade Federal do Pará. Metodologia: Trata-se de um estudo exploratório, prospectivo, qualitativo e quantitativo, do tipo corte-transverso, tendo como recorte temporal o período entre janeiro a junho de 2017. Resultado: Foram investigados 272 adolescentes, com idade média de 14 e 18 anos e renda familiar em torno de 1 a 3 salários mínimos, com consumo de pelo menos um medicamento nos últimos 30 dias; predominante uso de analgésicos e anti-inflamatórios não esteroidáis. Prevalência do consumo de álcool, tabaco, maconha, opiáceos, tranquilizantes para o gênero masculino e de álcool, opiáceos, tabaco, maconha, tranquilizantes para o gênero feminino. Observou-se uma prevalência global para o consumo de álcool associado ao consumo de outras drogas psicotrópicas. Conclusão: $O$ uso de substâncias psicoativas por adolescentes, um problema de saúde pública, requer atenção urgente, não apenas pelos efeitos diretos sobre estes, mas pelos diversos danos que gera na qualidade de vida das famílias, da comunidade e das demais pessoas envolvidas. Infere-se, assim, ser importante empoderar educadores sobre os riscos de consumo de medicamentos e de substâncias psicoativas na adolescência.

Palavras-chave: Adolescência. Uso de Medicamentos. Psicotrópicos. Drogas llícitas.

\begin{abstract}
Introduction: Adolescence is a developmental phase in which the first contacts with the use of psychoactive drugs, alcoholic beverages and other drugs are likely to occur. Objective: The aim was to know the profile of the use of drugs and psychoactive substances in high school adolescents enrolled in the School of Application of the Federal University of Pará. Methodology: Exploratory, prospective, qualitative and quantitative study, cross-sectional stud y, with a temporal cut between January and June 2017. Results: A total of 272 adolescents, mean age of 14 and 18 years, a family income between 1 and 3 minimum wages, consumption of, at least, one drug in the last 30 days, predominant use of analgesics and non-steroidal anti-inflammatory medicines. Prevalence of alcohol consumption, tobacco, marijuana, opiates, tranquilizers between men and prevalence of alcohol consumption, opiates, tobacco, marijuana, tranquilizers between female. There was a global prevalence of alcohol consumption associated with the use of other psychotropic drugs. Conclusion: The use of psychoactive substances by adolescents is a public health problem and requires urgent attention, not only for the direct effects on this use, but also for several damages in quality of life of families and community. It is also important to empower educators about the risks of drug and psychoactive substances use in adolescence.
\end{abstract}

Key-words: Adolescent. Drug Utilization. Psychotropic Drugs. Street Drugs.

Resumen. Introducción: La adolescencia es una fase de desarrollo en la cual tiende a ocurrir los primeros contactos con el uso de medicamentos psicoactivos, bebidas alcohólicas y otras drogas. Objetivo: El objetivo de esta investigación fue conocer el perfil de utilización de medicamentos y de sustancias psicoactivas en adolescentes inscritos en el nivel secundario de la Escuela de Aplicación de la Universidad Federal de Pará. Metodología: Se trata de un estudio exploratorio, prospectivo, cualitativo y cuantitativo, de tipo corte transversal, teniendo como recorte temporal el período entre enero y junio de 2017. Resultados: Fueron investigados 272 adolescentes, con edad media de 14 y 18 años y renta familiar en torno de 1 a 3 salarios mínimos, con consumo de por lo menos un medicamento en los últimos 30 días; predominante uso de analgésicos y antiinflamatorios no esteroideos. Prevalencia del consumo de alcohol, tabaco, marihuana, opiáceos, tranquilizantes para el género masculino y de alcohol, opiáceos, tabaco, marihuana, tranquilizantes para el género femenino. Se observó una prevalencia global para el consumo de alcohol asociado al consumo de otras drogas psicotrópicas. Conclusión: El uso de sustancias psicoactivas por adolescentes, un problema de salud pública, requiere atención urgente, no sólo por los efectos directos sobre éstos, sino por los diversos daños que genera en la calidad de vida de las familias, de la comunidad y de las demás personas involucradas. Se infiere, por lo tanto, ser importante empoderar a educadores sobre los riesgos de consumo de medicamentos y de sustancias psicoactivas en la adolescencia.

Palabras-clave: Adolescente. Utilización de Medicamentos. Psicotrópicos. Drogas llícitas. 


\section{Introdução}

Há diferentes problematizações acerca dos usos das drogas na sociedade contemporânea, resultado da antiga, e diversificada convivência das sociedades humanas com essas substâncias; hoje, ilícitas, cuja ação psicoativa é precisamente o que qualifica seu uso em rituais místicos, religiosos, lúdicos ou produtivos e criativos; por exemplo, por serem uma fonte de satisfação e prazer que potencializa o projeto humano de felicidade(1).

O uso de drogas é um fator de risco; ou seja, fonte de multiplicidades de fatores sociais e/ou pessoais, que torna o indivíduo vulnerável ao seu uso indevido. Comumente, essa prática de utilizar substâncias para alteração do nível de consciência tende a ter início na fase da adolescência(2, $3,4)$. A adolescência é uma fase do desenvolvimento humano - de transição entre a infância e a vida adulta -, na qual o indivíduo vivencia uma etapa evolutiva, única e exclusiva da espécie humana. Nesta fase acontecem intensas e profundas transformações físicas, mentais e sociais, que, inexoravelmente, o conduzirão a exibir as características de homem ou de mulher adultos(5).

Fase de novas sensações e experiências, a adolescência é considerada um período de risco com relação ao uso de substâncias psicoativas, uso irracional de medicamentos e danos associados a estes consumos $(4,6)$. Neste contexto, a educação está envolvida enquanto dimensão intrínseca às relações interpessoais e à vida social, o que coloca as experiências com as drogas como parte essencial dos processos de socialização e integração cultural.

O contexto social e a dinâmica familiar, no qual o adolescente está inserido, representa um papel fundamental nesta etapa da vida, fornecendo valores, regras e expectativas; bem como, os meios concretos para a viabilização de seu projeto de vida. $\mathrm{O}$ fato do adolescente não se sentir acolhido e não ter um bom relacionamento com a família, pode levá-lo a se envolver com o grupo de pares desviantes que fazem uso de substâncias ilícitas, colocando-o em risco de dependência química $(3,4)$.

A família é um lugar privilegiado para a promoção da educação. Mesmo que o jovem passe a conviver mais em outros ambientes como escola, clubes, shopping; entre outros, é no seio da família que os valores morais e os padrões de conduta são adquiridos. Quando esses valores não são adquiridos adequadamente durante a infância é que outros ambientes poderão ter influência de risco na adolescência(3, 4).

Estudos referentes ao uso de drogas por adolescentes convergem para o consenso de que famílias disfuncionais; ou seja, aquelas nas quais existe um funcionamento patológico com relação à comunicação, estabelecimento de regras e de limites e a falta de afeto, costumam ser o tipo mais encontrado em adolescentes dependentes de drogas(7). O uso de substâncias entre adolescentes vem provocando preocupação e, até mesmo, um alto custo econômico, por se tratar de um grupo vulnerável a este consumo.

Reconhece-se, que os pais servem de modelo para os adolescentes na experimentação e no início do contato com o álcool ou outras drogas. O uso de drogas pelos pais e outros familiares é certamente uma das grandes influências para que os adolescentes se tornem dependentes de drogas. Há, também, uma relação entre violência intrafamiliar e dependência de drogas na adolescência. Não apenas os adolescentes que vivenciam situações de abuso físico, psicológico ou social, mas o adolescente que frequentemente observa pais, irmãos, vizinhos em situações de violência(7).

A dependência química é resultante de um complexo sistema de aprendizagem, no qual o cérebro é reforçado pelo efeito psicoativo satisfatório, ativando os circuitos de recompensa, o que torna mais provável a repetição deste comportamento. Do mesmo modo, a dependência física está correlacionada, já que o sujeito repete o comportamento para interromper o desconforto da abstinência. Os padrões compulsivos na procura e consumo da droga, no entanto, são resultados de uma complexa aprendizagem que envolvem fatores psicológicos, neurológicos e sociais $(8,9)$.

Em adição, a problemática do uso irracional de medicamentos com poderes estimulantes e narcotizantes compõe um quadro de uma cultura farmacológica que valoriza a automedicação, justifica a importância de estudos que explorem esse cenário; principalmente em países onde não há um sistema de monitorização da utilização de medicamentos(9). O conceito de medicalização é atual e relevante para compreendermos alguns problemas da sociedade moderna.

Dados da United Nations (NU)(10), apontam para o crescimento do uso do Metilfenidato (anfetamina) e Clonazepam (ansiolítico), que tem sido prescrito para crianças e adolescentes no cenário brasileiro, em uma relação perversa no campo da medicalização e educação. Apesar de não figurar entre os 10 maiores consumidores mundiais per capita, a importação do Metilfenidato pelo Brasil apresentou aumento de mais de $300 \%$, passando de $578 \mathrm{~kg}$ importados em 2012 para $1.820 \mathrm{~kg}$ importados em 2013. Ainda, em 2013, o Brasil tornou-se líder na fabricação de Clonazepam, com 3,2 toneladas fabricadas no ano, tendo vendido em março 835.044 caixas.

A caracterização do padrão de utilização de medicamentos em uma comunidade é reconhecida pela World Health Organization (WHO)(11) como um importante indicador sanitário, contribuindo para melhor conhecer o modo como as populações utilizam os recursos terapêuticos. O uso de medicamentos atua de forma importante na prestação de cuidados da saúde, quando usado de forma racional, podendo ajudar a curar doenças e aliviar sintomas; porém, o seu uso irracional continua sendo um dos principais problemas enfrentado pelos órgãos de saúde no mundo(6).

Nesta direção, uma importante vertente é o conhecimento do uso de medicamentos e de substâncias psicoativas entre estudantes do ensino fundamental e médio, pois aí se incluem os adolescentes, população de alto risco para a drogadição. Reconhece-se que os adolescentes estão mais 
expostos ao uso de drogas, sendo que quanto mais precoce o início, piores serão as consequências $(12,13,14)$. A Política Nacional sobre Drogas no Brasil(15, 16), ressalta a relevância de estudos sobre o consumo de drogas lícitas e ilícitas. Assim sendo, este trabalho objetivou um delineamento transversal do perfil de consumo de medicamento e substâncias psicoativas em estudantes do ensino médio de uma escola no munícipio de Belém, PA.

\section{Metodologias}

Trata-se de um estudo transversal qualitativo e quantitativo $(17,18)$, tendo como recorte temporal o período de janeiro a junho de 2017. A amostra foi constituída por 272 alunos na faixa etária de 14 a 23 anos de idade, matriculados no período matutino na Escola de Aplicação da Universidade Federal do Pará. Para obtenção dos dados foi utilizado um formulário estruturado de autopreenchimento, sendo aplicado após os alunos terem sido esclarecidos sobre a problemática do uso irracional de medicamentos e de substâncias psicoativas, bem como sobre o objetivo do trabalho e a importância de responderem corretamente.

As variáveis investigadas foram: perfil socioeconômico, utilização de medicamentos pelos responsáveis, consumo de medicamentos e utilização de substâncias psicoativas pelos alunos, sendo estas: maconha, alucinógenos, cocaína, crack, ecstasy, anticolinérgico, solventes orgânicos, opiáceos, sedativos ou barbitúricos, tranquilizantes, anfetamínicos, anabolizantes, entre outras drogas.

Foi realizada uma análise descritiva das variáveis coletadas e os testes estatísticos de correlação. A análise de dados foi realizada através do programa BioEstat ${ }^{\circledR}$ (5.3), utilizando-se o Intervalo de Confiança de $95 \%$ para o teste do Qui-quadrado para avaliar as possíveis correlações entre as diferentes variáveis categóricas estudadas.

Em concordância com a Resolução do Conselho Nacional de Saúde n o 466, de 12 de dezembro de 2012, (19) que aprova as diretrizes e as normas que regulamentam as pesquisas envolvendo seres humanos no Brasil, esta investigação foi registrada na Plataforma Brasil sob CAAE n 61004516.3.0000.0018) e aprovada via Protocolo $n^{\circ} 106896 / 2016$.

\section{Resultados e discussão}

Dados socioeconômicos

Do total de 272 adolescentes do ensino médio entrevistados na Escola de Aplicação da Universidade Federal do Pará, na cidade de Belém, 30,88\% eram do $1^{\circ}$ ano; $43,38 \%$ do $2^{\circ}$ ano; $25,00 \%$ do terceiro e dois $(0,74 \%)$ alunos não responderam, sendo $38,24 \%$ do gênero masculino e $61,76 \%$ feminino. A faixa etária variou de 14 a 23 anos de idade, onde $97,43 \%$ tinham entre 14 e 18 anos e 2,57\% acima de 19 anos. Quanto a renda familiar, 37,50\% tinham renda de

\begin{tabular}{|c|c|c|}
\hline Variável & $\mathbf{n}$ & $\%$ \\
\hline \multicolumn{3}{|l|}{ Gênero } \\
\hline Masculino & 168 & 61.76 \\
\hline Feminino & 104 & 38.24 \\
\hline \multicolumn{3}{|l|}{ Idade } \\
\hline Mínima & 14 & - \\
\hline Média & 18.22 & - \\
\hline Máxima & 23 & - \\
\hline \multicolumn{3}{|l|}{ Série } \\
\hline $1^{\circ}$ ano & 84 & 30.88 \\
\hline $2^{\circ}$ ano & 118 & 43.38 \\
\hline $3^{\circ}$ ano & 68 & 25.00 \\
\hline \multicolumn{3}{|l|}{ Renda } \\
\hline 1 a 3 SM* $^{*}$ & 102 & 37.50 \\
\hline 4 a 6 SM & 52 & 19.12 \\
\hline Mais de 7 SM & 35 & 12.87 \\
\hline \multicolumn{3}{|l|}{ Dependentes } \\
\hline $1-2$ & 36 & 13.24 \\
\hline $3-5$ & 188 & 69.12 \\
\hline Acima de 6 & 40 & 14.71 \\
\hline \multicolumn{3}{|l|}{ Escolaridade do responsável } \\
\hline Fundamental & 12 & 4.41 \\
\hline Médio & 86 & 31.62 \\
\hline Superior & 118 & 43.38 \\
\hline
\end{tabular}

Tabela 1. Dados socioeconômicos dos alunos Escola de Aplicação da Universidade Federal do Pará em Belém (PA).

01 a 03 salários mínimos, 19,12\% de 04 a 06, 09,93\% de 07 a 10 e $02,94 \%$ mais de 10 salários mínimos, sendo que $30,51 \%$ não souberam informar (Tabela 1 ).

\section{Consumo de medicamentos nos últimos 30 dias}

Quanto ao consumo de medicamentos nos últimos 30 dias, $87,1 \%$ adolescentes haviam consumido pelo menos um medicamento. Quando inqueridos se seus pais e/ou responsáveis faziam uso contínuo de medicamentos, $84,93 \%$ responderam positivamente. Desses, observou-se que $70,22 \%$ dos filhos também faziam uso eventual de algum tipo de medicamento.

Os principais medicamentos utilizados pelos alunos e seus usos referidos estão apresentados no Quadro 1, onde se observa a prevalência do uso de analgésicos e antiinflamatórios não esteroidáis (AINE). Dos medicamentos utilizados, 36,57\% foi por prescrição médica, 28,70\% não foi informado, $17,13 \%$ foi indicado por parente ou pessoa próxima, $8,33 \%$ por auto-medicação, $5,56 \%$ por farmacêutico e $3,70 \%$ por atendente de farmácia e/ou dentistas. 


\begin{tabular}{|c|c|c|c|c|c|c|c|}
\hline Medicamento & Uso referido & $\mathbf{n}$ & $\%$ & Medicamento & Uso referido & $\mathbf{n}$ & $\%$ \\
\hline Dipirona & Analgésico & 52 & 21,90 & Alprazolam & Ansiolitico & 1 & 0,42 \\
\hline Paracetamol & Analgésico & 25 & 10,54 & Amoxicilina & Antibiótico & 1 & 0,42 \\
\hline Escopalamina & Antiespasmódico & 12 & 5,06 & Acebrofilina & Broncodilatador & 1 & 0,42 \\
\hline Dipirona sódica + Associações & AINE & 8 & 3,37 & Tiocolchicosídeo & $\begin{array}{c}\text { Relaxante } \\
\text { muscular }\end{array}$ & 1 & 0,42 \\
\hline Nimesulida & AINE & 8 & 3,37 & \begin{tabular}{|l|}
$\begin{array}{l}\text { Etinilestradiol + } \\
\text { ciproterona }\end{array}$ \\
\end{tabular} & Antiácne & 1 & 0,42 \\
\hline Ácido ascórbico & Antigripal & 7 & 2,95 & Escitalopram & Antidepressivo & 1 & 0,42 \\
\hline Ibuprofeno & Anti-inflamatório & 6 & 2,53 & Ambroxol & Expectorante & 1 & 0,42 \\
\hline $\begin{array}{l}\text { Paracetamol + clorfeniramina } \\
+ \text { fenilefrina }\end{array}$ & $\begin{array}{l}\text { Relaxante } \\
\text { muscular }\end{array}$ & 6 & 2,53 & Bisacodil & Laxante & 1 & 0,42 \\
\hline Ácido Acetilsalicilico & AINE & 4 & 1,68 & Metformina & Antidiabético & 1 & 0,42 \\
\hline Polivitamínicos & $\begin{array}{c}\text { Suplemento } \\
\text { alimentar }\end{array}$ & 4 & 1,68 & Naproxeno & AINE & 1 & 0,42 \\
\hline Isotretinoína & Antiácne & 4 & 1,68 & Dropropizina & Antitussigeno & 1 & 0,42 \\
\hline $\begin{array}{l}\text { Colina } \underset{\text { racemetionina }}{+} \text { betaína } \\
\text { ract }\end{array}$ & $\begin{array}{c}\text { Distúrbios } \\
\text { metabólicos }\end{array}$ & 3 & 1,26 & Omeprazol & Gastrite & 1 & 0,42 \\
\hline Cefalexina & Antibiótico & 2 & 0,84 & Olanzapina & Antipsicótico & 1 & 0,42 \\
\hline Diclofenaco & AINE & 2 & 0,84 & Pantoprazol & Gastrite & 1 & 0,42 \\
\hline Dimenidrinato & Antiemético & 2 & 0,84 & \begin{tabular}{|ll}
$\begin{array}{l}\text { Lansoprazol } \\
\text { laritromicina } \\
\text { amoxicilina }\end{array}$ & + \\
\end{tabular} & Antibiótico & 1 & 0,42 \\
\hline Loperamida & Antidiarreico & 2 & 0,84 & Dexclorfeniramina & $\begin{array}{c}\text { Anti- } \\
\text { histaminico }\end{array}$ & 1 & 0,42 \\
\hline Albendazol & Antiparasitário & 1 & 0,42 & $\begin{array}{l}\text { Ácido } \\
\text { mefenâmico }\end{array}$ & AINE & 1 & 0,42 \\
\hline Fexofenadine & Anti-histamínico & 1 & 0,42 & Imidazopiridina & Hipnótico & 1 & 0,42 \\
\hline Loratadina & Anti-histamínico & 1 & 0,42 & Benzetacil & Antibiótico & 1 & 0,42 \\
\hline \multicolumn{6}{|c|}{ Informaram ter usado medicamentos nos últimos 30 dias } & 237 & 87,1 \\
\hline \multicolumn{6}{|c|}{ Não informaram ter usado medicamentos nos últimos 30 dias } & 35 & 12,8 \\
\hline \multicolumn{6}{|c|}{ Pais ou responsáveis que fazem uso de medicamentos } & 201 & 84,93 \\
\hline \multicolumn{6}{|l|}{ Total de alunos entrevistados } & 272 & 100 \\
\hline
\end{tabular}

Fonte: os autores.

Quadro 1: Medicamentos utilizados pelos dos alunos Escola de Aplicação da Universidade Federal do Pará em Belém (PA) nos últimos 30 dias.

Utilização de medicamentos e correlação com sexo, idade, série escolar e renda familiar

A Tabela 02 apresenta as principais classes de medicamentos utilizados e suas possíveis correlações com as variáveis idade, grau escolar e renda familiar. Constatouse, ao analisar os resultados, que no aspecto global não há diferença significativa entre gênero, faixa etária e a série escolar dos entrevistados. Entretanto, nas classes dos analgésicos e dos antidepressivos foi observado que eram mais utilizados por aqueles que possuem menor renda. Morgan et $\mathrm{al}(20)$ e Bertoldi et al(21) registram que há prevalência de uso de medicamento pelo gênero feminino, sendo este fenômeno atribuído, entre outros fatores, ao uso de contraceptivos, assim como a um maior cuidado com a saúde e procura pelos serviços de saúde.

Sabe-se que o gasto familiar com saúde é um fator importante para a renda familiar; ou seja, quanto maior a renda, maior o gasto absoluto com saúde. No entanto, são as famílias com menor renda que comprometem, proporcionalmente, a maior parte de sua renda com esses gastos(20, 21).

De acordo com Viégas e Freire(22) o debate sobre a medicalização da vida tem ganhado corpo na produção científica brasileira, sobretudo a partir de 2010, fenômeno esse atribuído, dentre outros fatores, à fundação do Fórum sobre

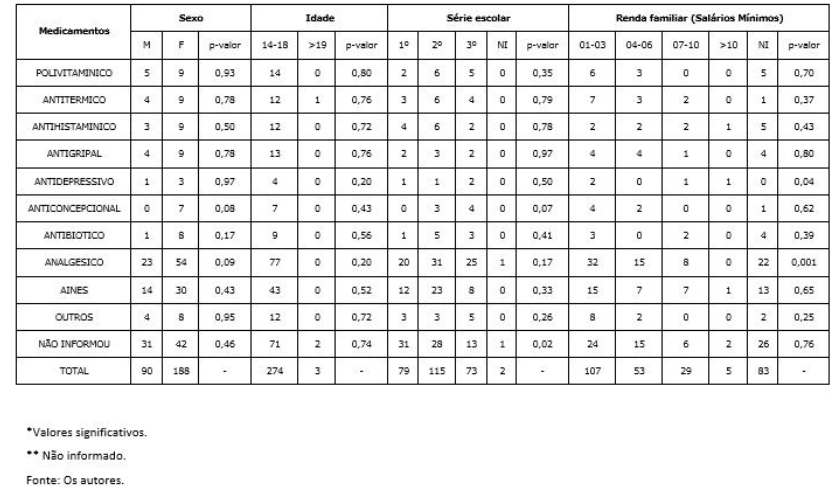

Tabela 2. Utilização de medicamentos e correlação com sexo, idade, série escolar e renda familiar.

Medicalização da Educação e da Sociedade; movimento social que assumiu como um de seus desafios a ampliação e qualificação desse debate no Brasil. Importante estratégia para detecção, análise e solução dos problemas relacionados ao uso irracional de medicamentos; em especial na adolescência, por se tratar de um grupo de risco para o uso de drogas lícitas e ilícitas, onde os fatores que podem levá-los a utilizá-las são variados e incluem a interação de múltiplos aspectos.

Para Oliveira, Harayama e Viégas(6), a medicalização envolve processos mais amplos que não se restringem ao medicamento, possuindo uma lógica mais sutil e perversa. Do ponto de vista histórico, é no final da década de 1960 que o termo medicalização surge como um importante instrumento teórico de denúncia da crescente intervenção política da medicina no corpo social, por meio do estabelecimento de normas morais de conduta e prescrição e proscrição de comportamentos, o que tornaria os indivíduos dependentes dos saberes produzidos pelos agentes educativo-terapêuticos(22).

A partir da década de 1970 houve uma tendência social de transformar as dores e questões da vida humana em temas de domínio médico, produzindo, como efeito, a perda da autonomia dos sujeitos(23). Estes autores reconhecem Michel Foucault como um estudioso do fenômeno da medicalização, o qual trouxe importantes críticas à constituição de uma sociedade na qual o indivíduo e a população são entendidos e manejados por meio da medicina. Patto(24), também, faz uma crítica à psicologização do fracasso escolar, explicitando o processo de medicalização da educação.

De acordo com o Fórum sobre Medicalização da Educação e da Sociedade(25), medicalização envolve um tipo de racionalidade determinista que desconsidera a complexidade da vida humana, reduzindo-a a questões de cunho individual, seja em seu aspecto orgânico, seja em seu aspecto psíquico, seja em uma leitura restrita e naturalizada dos aspectos sociais. Nessa concepção, características comportamentais são tomadas apenas a partir da perspectiva do indivíduo isolado, que passaria a ser o único responsável 
por sua inadaptação às normas e padrões sociais dominantes.

Há diferenças entre medicalização, patologização, psicologização, psiquiatrização e criminalização, conceitos que, embora tenham afinidade, não são sinônimos. No que concerne à definição propriamente dita, explicita que medicalização envolve uma forma de interpretar os fenômenos da vida que, aderida à ideologia dominante, desconsidera sua complexidade, reduzindo tudo a características individuais, não raro vistas como se fossem de determinação orgânica(26).

No que diz respeito à diferenciação, Gaudenzi e Ortega(26) explicitam que o fenômeno é mais amplo do que a prescrição (abusiva ou não) de medicamentos. Ou seja, há pessoas medicadas que não estão medicalizadas, assim como há pessoas medicalizadas que não fazem uso de medicamento. Isso sem falar no crescente mercado de "terapias alternativas", que, apesar de não prescrever medicamentos, ainda perpetua a lógica medicalizante.

A medicalização é terreno fértil para os fenômenos da patologização, da psiquiatrização, da psicologização e da criminalização das diferenças e da pobreza. O consumo de psicofármacos no Brasil parece ser importante sintoma da massificação da medicalização no país(7).

Utilização de substâncias psicoativas e correlação com gênero, idade, série escolar e renda familiar

Não foi observado diferença significativa entre o gênero, faixa etária e a série dos entrevistados quanto ao uso de medicamentos e substâncias psicoativas (Tabela 03). Observou-se, que os alunos com menor renda familiar foram aqueles que mais utilizavam substâncias psicoativas. Sabe-se que a baixa renda é um potencial gerador de fatores de risco quanto ao desenvolvimento psicossocial e uma possível utilização de droga. De acordo com o Centro Brasileiro de Informações sobre Drogas Psicotrópicas (CEBRID)(27), há estudos que correlacionam a renda familiar, o nível socioeconômico e o uso de substâncias psicoativas. Adolescentes de classe média e alta que se encontram em processo de amadurecimento cognitivo e emocional, apresentam uma tendência maior de uso dessas substâncias, em especial para o uso de álcool e tabaco $(28,29,30)$.

Destaca-se, aqui, os achados para os alunos do gênero masculino do $2^{\circ}$ ano, onde foi observado a prevalência do consumo de álcool, tabaco, maconha, opiáceos, tranquilizantes, entre outros psicotrópicos; assim como, para as alunas do gênero feminino do $2^{\circ}$ ano, há uma prevalência do uso álcool, opiáceos, tabaco, maconha, tranquilizantes, entre outros psicotrópicos. Contudo, a utilização de maconha, álcool e tabaco foram predominantes, o que está em concordância com os resultados divulgados pelo Centro Brasileiro de Informações sobre Drogas Psicotrópicas (CEBRID)(27), que afirma que adolescentes de 16 a 18 anos são, comumente, os que apresentam maior uso de substância psicotrópicas entre os estudantes.

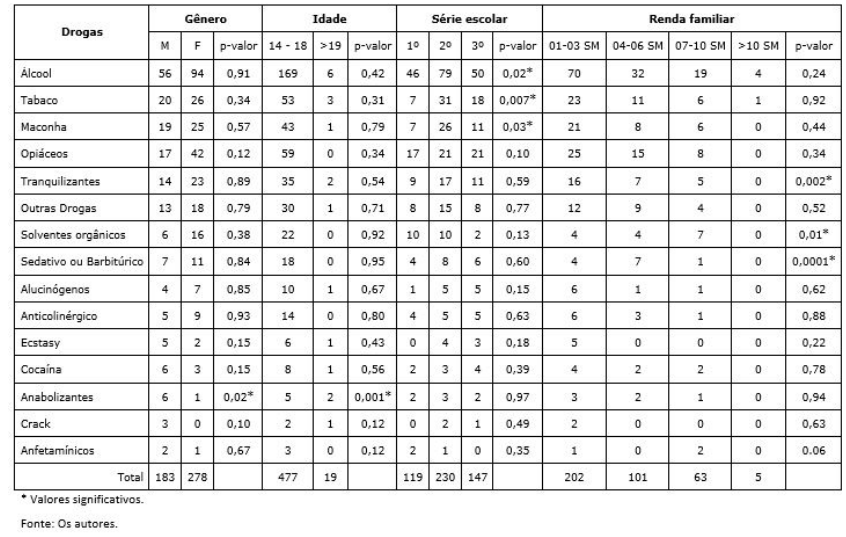

Tabela 3. Utilização de medicamentos e substâncias psicoativas e a correlação com gênero, idade, série escolar e renda familiar.

Associação entre uso de álcool e substâncias psicoativas

Observou-se uma prevalência global para o consumo de álcool associado ao consumo de opiáceos, maconha, tranquilizantes, outras drogas psicotrópicas, solventes orgânicos, sedativo/barbitúrico (Tabela 4). Isto pode ser explicado pelo fato dos jovens utilizarem as drogas como um passaporte para a alegria, uma forma de relaxar e se divertir, acreditando não ser perigoso, sendo que muitos nem o consideram uma droga e muito menos as consequências do seu consumo, mas que em alguns casos é a porta de entrada para o uso de drogas de abuso.

Estudos apontam que o dano gerado pela exposição às substâncias psicotrópicas na adolescência pode refletir no abandono escolar e do lar, baixo rendimento na aprendizagem, problemas de saúde e comportamentos de risco à saúde, como insônia e falta de apetite, baixa autoestima, ideação e comportamento suicida e comportamento violento $(27,28,29,30)$.

Registra-se, que são necessárias investigações mais abrangentes sobre os aspectos envolvidos no consumo de medicamentos e de substâncias psicoativas entre os estudantes, bem como sobre os impactos deste consumo no processo de ensino-aprendizagem e sobre as consequências físicas, psíquicas e sociais para o estudante. Embora os resultados aqui apresentados sejam representativos para o local da pesquisa, é limitado quando se faz referência à população de adolescentes em âmbito regional e nacional. Estudos mais abrangentes são oportunos e necessários.

\section{Conclusões}

Evidências apontam que na Escola de Aplicação da Universidade Federal do Pará, em Belém (PA), há uso de medicamentos e substâncias psicoativas, destacando-se como achados:

- Do total de 272 adolescentes matriculados no ensino médio: $30,88 \%$ eram do $1^{\circ}$ ano; $43,38 \%$ do $2^{\circ}$ ano; $25,00 \%$ do 


\begin{tabular}{|l|c|c|c|}
\hline \multirow{2}{*}{ Drogas } & \multicolumn{3}{|c|}{ Álcool } \\
\cline { 2 - 4 } & Usa & Não & p-valor \\
\hline Opiáceos & 45 & 83 & $0,04^{*}$ \\
\hline Maconha & 43 & 96 & $0,0001^{*}$ \\
\hline Tranquilizantes & 33 & 93 & $0,001^{*}$ \\
\hline Outras Drogas & 25 & 91 & 0,06 \\
\hline Solventes orgânicos & 15 & 90 & 0,87 \\
\hline Sedativo / Barbitúrico & 14 & 93 & 0,32 \\
\hline Anticolinérgico & 11 & 94 & 0,39 \\
\hline Alucinógenos & 11 & 97 & $0,02^{*}$ \\
\hline Cocaína & 9 & 97 & $0,05^{*}$ \\
\hline Ecstasy & 7 & 97 & 0,11 \\
\hline Anabolizantes & 7 & 97 & 0,11 \\
\hline Crack & 3 & 97 & 0,48 \\
\hline Anfetamínicos & 3 & 97 & 0,48 \\
\hline
\end{tabular}

*Valores Significativo.

Fonte: Os autores.

Tabela 4. Associação global entre uso de álcool e substâncias psicoativas.

terceiro, onde prevalece o gênero feminino com 61,76\%. Quanto a idade, 97,43\% tinham entre 14 e 18 anos. Quanto a renda familiar $37,50 \%$ tinham entre 01 a 03 salários mínimos.

- $87,1 \%$ adolescentes haviam consumido pelo menos um medicamento nos últimos 30 dias. $84,93 \%$ dos pais e/ou responsáveis faziam uso cotidiano de medicamentos, dentre os quais $70,22 \%$ dos filhos também faziam eventual uso de algum tipo de medicamento.

- Dos medicamentos utilizados $36,57 \%$ foi por prescrição médica, 28,70\% não foi informado, 17,13\% foi indicado por parente ou pessoa próxima, $8,33 \%$ por auto-medicação, 5, $56 \%$ por farmacêutico e $3,70 \%$ por atendente de farmácia e/ou dentistas.

- Entre os principais medicamentos utilizados e seus usos referidos há uma prevalência do uso de analgésicos e antiinflamatórios não esteroidáis (AINE). Não foi observado diferença significativa entre o gênero, faixa etária e a série dos entrevistados e o uso de medicamentos e drogas psicoativas. Os alunos com menor renda familiar foram os que mais utilizam analgésicos e antidepressivos.

- Entre o gênero masculino do $2^{\circ}$ ano, foi observado a prevalência do consumo de álcool, tabaco, maconha, opiáceos, tranquilizantes, entre outros psicotrópicos. Entre o gênero feminino do $2^{\circ}$ ano, houve uma prevalência do uso de álcool, opiáceos, tabaco, maconha, tranquilizantes, entre outros psicotrópicos.

- Houve uma prevalência global para o consumo de álcool associado ao consumo de opiáceos, maconha, tranquilizantes, outras drogas psicotrópicas, solventes orgânicos, sedativo/barbitúrico.

Sabe-se, por meio de estudos científicos, que o uso de medicamentos e substâncias psicoativas adquire grande expressão entre os adolescentes, devido a impulsividade que marca esta etapa da vida.

Mundialmente, nacionalmente, regionalmente e localmente, o uso de medicamentos e de substâncias psicoativas por adolescentes, tem-se tornado um problema de saúde pública que requer atenção urgente, não apenas pelos efeitos diretos sobre estes, mas pelos diversos danos que gera na qualidade de vida das famílias, da comunidade e das demais pessoas envolvidas.

Reconhece-se que a escola - um espaço privilegiado para a prevenção, identificação precoce e intervenção sobre o consumo de medicamentos e de substâncias psicoativas carece de fortalecimento na articulação entre as estratégias de educação e de atenção à saúde do adolescente, sendo fundamental estimular o diálogo sobre o fenômeno do uso de medicamentos e substâncias psicoativas e suas reais consequências.

Infere-se, assim, ser importante empoderar educadores sobre os riscos de consumo de medicamentos e de substâncias psicoativas na adolescência; quiçá, sensibilizando-os ao permanente diálogo sobre a necessidade de adoção de condutas de estímulo à autoestima do adolescente, seja por meio da prática de esportes e de outras atividades que promovam o autoconhecimento, o lazer e a recreação.

\section{Referências}

1. Silva ML, Brandão B, Ribeiro TM. Drogas, medicalização e educação: Conquistas e ampliação dos espaços de discussão, ensino e pesquisa. Revista Teias. 2016; 17(45):17. In: Drogas, Medicalização e Educação. ISSN 1518-5370 [impresso]; 1982-0305 [eletrônico]. DOI: 10.12957/teias.

2. Sodelli MA. Abordagem proibicionista em desconstrução: compreensão fenomenológica existencial do uso de drogas. Revista Ciências e Saúde Coletiva. 2010; 15(3):637-644. ISSN 1413-8123.

3. Costa COM, Matos AM, Carvalho RC, Amaral MTR, Cruz NLA, Lopes TC. Uso frequente e precoce de bebidas alcoólicas na adolescência: análise de fatores associados. Adolesc Saude. 2013; 10(4):25-32.

4. Silva CMDA. O uso de drogas na adolescência: identificação de estratégias e proposta de prevenção. Trabalho de Conclusão de Curso apresentado ao curso de Especialização em Atenção Básica em Saúde da Família. Orientadora Profa. Dra. Erika de Cássia Lopes Chaves. Universidade Federal de Alfenas. Alfenas. MG. 2014.

5. Azevedo TDPL, Alves ED. Qualidade de vida de adolescentes: revisão da literatura e perspectivas atuais. Rev. Gest.Saúde (Brasília). 2016; 07(2):851-72. ISSN: 1982-4785. 
6. Oliveira EC, Harayama RM, Viégas LS. Drogas e medicalização na escola: Reflexões sobre um debate necessário. Revista Teias. 2016; 17(45):99-118. In: Drogas, Medicalização e Educação. ISSN 1518-5370 [impresso]; 1982-0305 [eletrônico]. DOI: 10.12957/teias.

7. Acselrad G, (Org.). Quem tem medo de falar sobre drogas? Saber mais para proteger. Rio de Janeiro: Editora FGV, 2015. 164p. ISBN: 978-85-225-1629-2.

8. Organização Mundial da Saúde (OMS). Neurociências: consumo e dependência de substâncias psicoativas (Resumo). Genebra: OMS, 2004.

9. Ofori-Asenso R, Brhlikova P, Pollock AM. Prescribing indicators at primary health care centers within the WHO African region: a systematic analysis (1995-2015). BMC Public Health. 2016; 16(724):12-14. DOI: 10.1186/s12889-016-3428-8.

10. United Nations (NU). Report of the International Narcotics Control Board for 2014. United Nations: International Narcotics Control Board, January 2015.

11. World Health Organization (WHO). The Rational Use of Drugs. Report of the Conference of Experts. Geneva: WHO; 1987.

12. Teixeira LA, Edler FC. História e cultura da medicina no Brasil. São Paulo: AORI Produções Culturais, 2012.

13. Temporão JG. A propaganda de medicamentos e o mito da saúde. Rio de Janeiro: Edições Graal, 1986.

14. Torcato CE. O metilfenidato, a escola e a cultura farmacológica contemporânea. Revista Teias. 2016; 17(45):83-97. In: Drogas, Medicalização e Educação. ISSN 1518-5370 [impresso]; 1982-0305 [eletrônico]. DOI: $10.12957 /$ teias.

15. Brasil. Conselho Nacional Antidrogas (CONAD). Resolução n³/GSIPR/CH/CONAD de 27 de outubro de 2005. Aprova a Política Nacional sobre Drogas. Brasília. DF.

16. Brasil. Ministério da Justiça. Legislação e políticas públicas sobre drogas no Brasil. Brasília: Ministério da Justiça, Secretaria Nacional de Políticas sobre Drogas, 2011. $106 \mathrm{p}$.

17. Minayo MCS. Análise qualitativa: teoria, passos e fidedignidade. Ciênc. Saúde Coletiva, 2012; 17(3): 621-6.

18. Turato ER. Métodos qualitativos e quantitativos na área da saúde: definições, diferenças e seus objetos de pesquisa. Rev Saúde Pública, 2005; 39(3): 507-14.

19. Brasil. Conselho Nacional de Saúde. Resolução n o 466 de 12 de dezembro de 2012. Aprova as diretrizes e normas regulamentadoras de pesquisas envolvendo seres humanos. Brasília, 2012.

20. Morgan TK, Williamson M, Pirotta M, Stewart K, Myers SP, Barnes J. A national census of medicines use: a 24-hour snapshot of Australians aged 50 years and older. Med J Aust. 2012; 196(1):50-3. DOI:10.5694/mja11.10698

21. Bertoldii AD. Perfil sociodemográfico dos usuários de medicamentos no Brasil: resultados da PNAUM 2014. Revista Saúde Pública, 2016.

22. Viégas LS, Freire KES. O debate sobre medicalização na psicologia escolar e educacional em uma perspectiva crítica: aspectos históricos. In: DANTAS, J.B. (Org.). A infância medicalizada: discursos, práticas e saberes para o enfrentamento da medicalização da vida. Curitiba: CRV Editora, 2015. p. 103-122.

23. Gonzaga JP, Martins SA, Araújo ESA, Baltar SLSMA. Conhecimento dos estudantes do ensino médio de Santana do Ipanema sobre os esteróides anabolizantes andrógenos. Ensino, Saúde e Ambiente. 2012; 5(2): 45-56.

24. Patto, MHS. A produção do fracasso escolar: histórias de submissão e rebeldia. São Paulo: T.A. Queiroz, 1990.

25. Fórum sobre Medicalização da Educação e da Sociedade. Carta do IV Seminário Internacional a Educação Medicalizada: desver o mundo, perturbar os sentidos. Salvador, Bahia, 2015.

26. Gaudenzi P, Ortega F. O estatuto da medicalização e as interpretações de Ivan Illich e Michel Foucault como ferramentas conceituais para o estudo da desmedicalização. Interface (Botucatu). 2012; 16(40):21-34. ISSN 1807-5762. http://dx.doi.org/10.1590/S1414-32832012005000020.

27. Centro Brasileiro de Informações sobre Drogas Psicotrópicas (CEBRID). VI Levantamento Nacional sobre o Consumo de Drogas Psicotrópicas entre Estudantes do Ensino Fundamental e Médio das Redes Pública e Privada de Ensino nas 27 Capitais Brasileiras. Carlini EA. (supervisão) et. al. Universidade Federal de São Paulo (UNIFESP). Secretaria Nacional de Políticas sobre Drogas (SENAD). Brasília, 2010. 503 p. ISBN: 978-85-60662-63-0.

28. Andrade AG, Duarte PCAV, Oliveira LG. Levantamento Nacional sobre o Uso de Álcool, Tabaco e Outras Drogas entre Universitários das 27 Capitais Brasileiras. Faculdade de Medicina da Universidade de São Paulo - USP e SENAD (Secretaria Nacional de Políticas sobre Drogas). 2010.

29. Lopes AP, Rezende MM. Consumo de substâncias psicoativas em estudantes do ensino médio. Psicol. teor. prat. $2014 ; 16(2): 29-40$.

30. Silva MVS, Trindade JBC, Oliveira CC, Mota GS, Carnielli L, Silva MFJ, Andrade MA. Consumo de medicamentos por estudantes adolescentes de Escola de Ensino Fundamental do município de Vitória. Rev Ciênc Farm Básica Apl.,2009;30(1):99-104. ISSN 1808-4532. 